\title{
FROM IMMERSIONS TO EMBEDDINGS OF SMOOTH MANIFOLDS
}

BY

FRANCIS X. CONNOLLY

Introduction. In this paper we study the question of when an immersion between two smooth manifolds, $f: V \rightarrow M$, is homotopic to an embedding. We introduce an invariant $\gamma(f)$ which determines, under suitable connectivity hypotheses, whether $f$ is homotopic to an embedding or not. Specifically, if $V^{n}$ is $k / 2$ connected, $M^{m} k$-connected and $m>2 n-k$, our main theorem (3.5) shows that $f$ is homotopic to an embedding if $\gamma(f)=0, m \geqq(3 / 2)(n+1)$, and the normal bundle to the immersion has a cross section. Thus the connectivity of $V$ is about half of what is usually required.

The invariant $\gamma(f)$ is usually readily computed, since there is a simple formula for it in terms of Poincaré duality on $V$ and $M$, the fundamental class of $V$, and the maps $f^{*}$ and $f_{*}$ (see $2.8,2.10$ ). In particular it is always zero for $M=R^{m}(4.2)$. This yields essentially the results of deSapio [2] immediately. For the standard embedding questions about $\mathrm{CP}^{n}$ and $\mathrm{HP}^{n}$ it yields (using 4.3):

(i) If $\mathrm{CP}^{n}$ immerses in $R^{4 n-3}$, then it embeds in $R^{4 n-2}$.

(ii) If $\mathrm{HP}^{n}$ immerses in $R^{8 n-j}$, it embeds in $R^{8 n-j+1}$ for $j<8$.

Actually, the method gives more interesting results when $M \neq R^{m}$. For example if $V$ and $M$ are products of spheres (with correct connectivity) one can pretty nearly classify which continuous maps are homotopic to embeddings. Such special cases are developed at the end of Chapter 4.

As for notation, $v$ always denotes a normal bundle, $V_{x}$ denotes the tangent space of $V^{n}$ at $x$. Throughout the whole paper, if $f: V^{n} \rightarrow M^{m}$ is an immersion, the letter $r$ is reserved exclusively for $2 n-m$, the dimension of the double point set. All manifolds and immersions will be smooth (i.e. $C^{\infty}$ ) unless expressly referred to as piecewise linear.

I. Generalities on generic immersions.

1.1. Definition. Let $M^{m}$ and $V^{n}$ be smooth manifolds. An immersion $f: V \rightarrow M$ is generic if, for each $x_{1} \cdots x_{k}$ in $V$ with $f\left(x_{j}\right)=p$ for all $j$, then

$$
f_{*}\left(V_{x_{1}}\right)+\left\{\bigcap_{j=2}^{k} f_{*}\left(V_{x_{j}}\right)\right\}=M_{p}
$$

We will say that two generic immersions $f_{i}: V_{i} \rightarrow M, i=1,2$, intersect generically if the induced immersion of the disjoint union $f_{1} \cup f_{2}: V_{1} \cup V_{2} \rightarrow M$ is generic.

Received by the editors October 15, 1968.

Copyright (C) 1970, American Mathematical Society 
The following facts about generic immersions are more or less well known, or at least easily verified by standard methods.

1.2. The generic immersions are open and dense in $\operatorname{Imm}(V, M)$. (See [12].)

1.3. If $f_{i}: V_{i} \rightarrow M$ are generic immersions, $i=1$, 2, with $f_{1}\left(V_{1}\right)=f_{2}\left(V_{2}\right)$, and with $\operatorname{dim}(M)>\operatorname{dim}\left(V_{i}\right), i=1,2$, then there is a unique $C^{0}$ map $h: V_{1} \rightarrow V_{2}$ with $f_{1}=f_{2} h$, and this map is a diffeomorphism. (This is easily seen by noting that a point $x$ in $V_{1}$ is determined by its image $f_{1}(x)$, together with the image of its tangent plane.)

1.4. If $f: V \rightarrow M$ is a generic immersion, then the smooth map

$$
f \times f: V \times V-\Delta V \rightarrow M \times M
$$

is $t$-regular to the submanifold $\Delta M$ in $M \times M$. If $f_{i}: V_{i} \rightarrow M, i=1,2$, are generically intersecting immersions, then $f_{1} \times f_{2}: V_{1} \times V_{2} \rightarrow M \times M$ is $t$-regular to $\Delta M$.

1.5. Definition. If $f: V^{n} \rightarrow M^{m}$ is a generic immersion, and

$$
\tilde{\chi}(f)=\{(x, y) \in V \times V-\Delta V: f(x)=f(y)\},
$$

then $\tilde{\chi}(f)$ is a smooth closed submanifold (by 1.4) of $V \times V$, called the double point manifold. Its dimension is therefore $2 n-m$; that is to say $\operatorname{dim} \tilde{\chi}(f)=r$. Also the map $\sigma: \tilde{\chi}(f) \rightarrow \tilde{\chi}(f)$ defined by $\sigma(x, y)=(y, x)$ is a smooth fixed point free involution. The quotient space $\tilde{\chi}(f) / \sigma$ is denoted $\chi(f)$ and is called the self intersection manifold; it is a smooth manifold and the quotient map $\pi: \tilde{\chi}(f) \rightarrow \chi(f)$ is a smooth double covering map. The map $\tilde{j}: \tilde{\chi}(f) \rightarrow V$ given by $j(x, y)=x$ is smooth, and there is a unique smooth map $j: \chi(f) \rightarrow M$ with $j \circ \pi=f \circ \tilde{j}$. We write $\tilde{X}(f)=\tilde{j}(\tilde{\chi}(f))$ and $X(f)=j(\chi(f))$; these are called the double point sets and self intersection sets respectively. Note $\tilde{X}(f)=\left\{x \in V: f^{-1} f(x) \neq x\right\}$, and $X(f)=f(\tilde{X}(f))$. Finally we write $\tilde{Y}(f)=\left\{x \in V: f^{-1} f(x)\right.$ contains at least 3 points $\}$.

Similarly, if $f_{i}: V_{i} \rightarrow M, i=1,2$, are generically intersecting immersions, we let $V=V_{1} \cup V_{2}, f=f_{1} \cup f_{2}$ (disjoint union) so $V \times V=V_{1} \times V_{1} \cup V_{1} \times V_{2} \cup V_{2} \times V_{1}$ $\cup V_{2} \times V_{2}$. We write $\chi\left(f_{1}, f_{2}\right)$ for $\tilde{\chi}(f) \cap V_{1} \times V_{2}, \chi\left(f_{2}, f_{1}\right)$ for $\tilde{\chi}(f) \cap V_{2} \times V_{1}$, $\tilde{\chi}\left(f_{1}, f_{2}\right)$ for $\chi\left(f_{1}, f_{2}\right) \cup \chi\left(f_{2}, f_{1}\right)$; we write $k\left(f_{1}, f_{2}\right)$, or just $k$, for the smooth map $\tilde{j}(f): \chi\left(f_{1}, f_{2}\right) \rightarrow V_{1}$, and we write $X\left(f_{1}, f_{2} ; V_{1}\right)$ for $\operatorname{Im}(k), X\left(f_{1}, f_{2}\right)$ for $\operatorname{Im}\left(f_{1} k\right)$ which is $f_{1}\left(V_{1}\right) \cap f_{2}\left(V_{2}\right)$. We write $\tau: \chi\left(f_{1}, f_{2}\right) \approx \chi\left(f_{2}, f_{1}\right)$ for $\sigma(f) \mid \chi\left(f_{1}, f_{2}\right)$ and we define $k^{\prime}\left(f_{1}, f_{2}\right): \chi\left(f_{1}, f_{2}\right) \rightarrow V_{2}$ by $k^{\prime}=j(f) \circ \tau$.

We also need two further results on generic immersions:

1.6. LeMMA. Let $f: V^{n} \rightarrow M^{m}$ be a generic immersion. Then there is a Riemannian metric on $M$ such that:

(a) If $(x, y) \in \tilde{\chi}(f)$, then $\left\{f_{*}\left(V_{x}\right)\right\}^{\perp} \subset f_{*}\left(V_{y}\right)$.

(b) $f(V)$ is totally geodesic in $M$ (i.e. geodesics of $M$ starting in and tangent to $f(V)$ stay in $f(V)$ ).

Proof. We first work locally. For any point $p$ in $M$, with $f^{-1}(p)=\left\{x_{1} \cdots x_{k}\right\}$, choose neighborhoods $\mathscr{U}_{i}$ of $x_{i}$ and $\mathscr{U}$ of $p$ which satisfy: 
(i) For each $i, f\left(\mathscr{U}_{i}\right)=\varphi_{i}^{-1}(0)$ for some smooth map $\varphi_{i}: \mathscr{U} \rightarrow R^{m-n}$ with rank $m-n$ everywhere.

(ii) The set $N=\bigcap_{i=1}^{k} f\left(\mathscr{U}_{i}\right)$ is a submanifold of $\mathscr{U}$ (necessarily, by 1.1 , having dimension $m-k(m-n))$ and there is a smooth map $\varphi_{0}: \mathscr{U} \rightarrow R^{s}, s=m-k(m-n)$, such that $\varphi_{0} \mid N$ is an embedding, and $\varphi_{0}(p)=0$.

Consider the map $\Phi=\varphi_{0} \times \varphi_{1} \times \cdots \times \varphi_{k}: \mathscr{U} \rightarrow R^{s} \times R^{m-n} \times \cdots \times R^{m-n}=R^{m}$. By 1.1 we see that $\bigcap_{i=1}^{k} f_{*}\left(V_{x_{i}}\right)=N_{p}$. But since $f_{*}\left(V_{x_{i}}\right)=\operatorname{Ker}\left(d \varphi_{i}\right)_{p}$ and $\operatorname{Ker}\left(d \varphi_{0}\right)_{p}$ $\cap N_{p}=0$, it follows that $\operatorname{Ker}(d \Phi)_{p}=0$, so that if $\mathscr{U}$ is chosen small enough we can assume $\Phi$ maps $\mathscr{U}$ diffeomorphically onto $N(0, \varepsilon)$, the $\varepsilon$ neighborhood of 0 in $R^{m}$. Clearly then, if $K_{i}$ is the orthogonal complement of the $i$ th copy of $R^{m-n}$ in $R^{m}$ as above, $\Phi$ maps $f\left(\mathscr{U}_{i}\right)$ onto $N(0, \varepsilon) \cap K_{i}$.

Now under the ordinary metric $g$ on $R^{m}$, it is obvious that the subspaces $K_{i}$ are totally geodesic and satisfy $K_{i}^{\perp} \subset K_{j}$ if $i \neq j$, and so if $q \in K_{i} \cap K_{j}$, we get $T\left(K_{i}\right)_{q}^{\perp}$ $\subset T\left(K_{j}\right)_{q}$. But let $g^{\prime}$ be the tensor $g^{\prime}\left(v_{x}, w_{x}\right)=\lambda\left(\|x\|^{2}\right) g\left(v_{x}, w_{x}\right)$, where $\lambda \geqq 0$. Under this "metric" we still have $T\left(K_{i}\right)_{q}^{\perp} \subset T\left(K_{j}\right)_{q}$ if $q \in K_{i} \cap K_{j}, i \neq j$, and $\lambda(q)>0$. Also, each $K_{i}$ is still totally geodesic under $g^{\prime}$ for it is easy to see that straight lines through 0 will still be paths of minimal length under $g^{\prime}$, and $K_{i}$ is thus the union of the geodesics through 0 which are tangent to a given subspace of the tangent space to $R^{m}$ at 0 .

Hence we can choose an appropriate $\lambda$ so that $\lambda$ is $>0$ only on $N(0, \varepsilon / 2)$, and then, choosing $\mathscr{U}^{\prime}=\Phi^{-1}(N(0, \varepsilon / 2))$ and pulling this metric $g^{\prime}$ back to $\mathscr{U}^{\prime}$, we see that we have proved:

For each point $p$ in $M$ there is a neighborhood $\mathscr{U}^{\prime}(p)$ and a metric $g^{\prime}$ on $\mathscr{U}^{\prime}$ which satisfies (a) and (b) of 1.6 for $f: f^{-1}\left(\mathscr{U}^{\prime}\right) \rightarrow \mathscr{U}^{\prime}$. Furthermore $g^{\prime}$ has a smooth extension to a tensor on all of $M$ by letting $g^{\prime}\left(v_{x}, w_{x}\right)=0$ if $x$ is not in $\mathscr{U}^{\prime}$.

To complete the proof, let $\left\{\mathscr{U}_{\alpha}\right\}$ be a locally finite cover of $M$ by such neighborhoods, and let $g_{\alpha}$ be the corresponding metrics. Consider the metric on $M$ given by $g=\sum_{\alpha} g_{\alpha}$. It is immediate that $g$ satisfies (a) of 1.6 for $f: V \rightarrow M$. Also if a path is a geodesic for $g_{\alpha}$ and for $g_{\beta}$ (i.e. it minimizes lengths) then this path also minimizes lengths for $g_{\alpha}+g_{\beta}$. It follows immediately that $g$ satisfies (b) of 1.6 for $f: V \rightarrow M$. This proves 1.6.

Note that an immersion $f: V \rightarrow M$ which satisfies $1.6($ a) must be generic since, if $x_{1} \cdots x_{k} \in f^{-1}(p)$, then $\left\{f_{*}\left(V_{x_{1}}\right)\right\}^{\perp} \subset f_{*}\left(V_{x_{j}}\right)$ for $j=2, \ldots, k$, so that

$$
M_{p}=f_{*}\left(V_{x_{1}}\right)+\left\{f_{*}\left(V_{x_{1}}\right)\right\}^{\perp} \subset f_{*}\left(V_{x_{1}}\right)+\bigcap_{j=2}^{k} f_{*}\left(V_{x_{j}}\right) .
$$

1.7. LEMMA. (A) Let $f: V^{n} \rightarrow M^{m}$ be a generic immersion. Then the map $\tilde{J}: \tilde{\chi}(f) \rightarrow V$ is a generic immersion and $\tilde{Y}(f)$ is its self intersection set (i.e. $\tilde{Y}(f)$ $=X(\tilde{j}))$. Furthermore, there is a canonical bundle equivalence $\lambda: \sigma^{*} \nu(\tilde{j}) \approx \tilde{j}^{*} \nu(f)$.

(B) Let $f_{i}: V_{i} \rightarrow M$ be generically intersecting immersions, $i=1,2$. Then the map $k: \chi\left(f_{1}, f_{2}\right) \rightarrow V_{1}$ is a generic immersion which intersects generically with $\tilde{j}: \tilde{\chi}\left(f_{1}\right) \rightarrow V_{1}$. Furthermore there is a canonical bundle equivalence $\lambda^{\prime}: \nu(k) \approx k^{\prime *} \nu\left(f_{2}\right)$. 
Proof. (B) is clearly a restatement of (A) along the lines of the second paragraph of 1.5 , so we prove (A) only.

We first show that $\tilde{j}$ is an immersion. Note that if $(x, y) \in \tilde{\chi}$, then a tangent vector $(v, w)$ in $V_{x} \times V_{y}$ is in the tangent space $\tilde{\chi}_{(x, y)}$ iff $(f \times f)_{*}(v, w)$ is tangent to $\Delta M$. So $\tilde{\chi}_{(x, y)}=\left\{(v, w) \in V_{x} \times V_{y}: f_{*}(v)=f_{*}(w)\right\}$. Thus $(f \circ \tilde{J})_{*} \tilde{\chi}_{(x, y)}=f_{*}\left(V_{x}\right) \cap f_{*}\left(V_{y}\right)$. By 1.1 this last space has dimension $\operatorname{dim} V_{x}+\operatorname{dim} V_{y}-\operatorname{dim} M=2 n-m=\operatorname{dim} \tilde{\chi}_{(x, y)}$. So $(f \circ \tilde{j})_{*}$ is $1-1$ and so $\tilde{j}_{*}$ is too. So $\tilde{j}$ is an immersion.

Now, to show $\sigma^{*} \nu(\tilde{j}) \approx \tilde{j}^{*} \nu(f)$, assume a Riemannian metric satisfying 1.6 has been chosen for $M$. It induces a metric on $V$. Now, if $(x, y) \in \tilde{\chi}(f)$, then the fiber $\left\{\sigma^{*} \nu(\tilde{j})\right\}_{(x, y)}$ is $\left\{\tilde{j}_{*} \tilde{\chi}_{(y, x)}\right\}^{\perp}$ in $V_{y}$ and this is just $\left\{v \in V_{y}: f_{*}(v) \in\left(f_{*} V_{x}\right)^{\left.\perp \subset M_{p}\right\}}\right.$ since $f_{*}$ preserves inner products. But by $1.6(\mathrm{a})\left(f_{*} V_{x}\right)^{\perp} \subset f_{*} V_{y}$, so the fiber $\left\{\tilde{j}^{*} \nu(f)\right\}_{(x, y)}$ $=\nu(f)_{x}=\left(f_{*} V_{x}\right)^{\perp}$ is just $f_{*}\left(\sigma^{*} \nu(j)_{(x, y)}\right)$. Thus the map

$$
f_{*}:\left\{\sigma^{*} \nu(\tilde{j})\right\}_{(x, y)} \rightarrow\left\{\tilde{J}^{*} \nu(f)\right\}_{(x, y)}
$$

gives the desired bundle equivalence, $\lambda: \sigma^{*} \nu(\tilde{j}) \approx \tilde{j}^{*} \nu(f)$.

Finally if $\left(x, y_{1}\right)$ and $\left(x, y_{2}\right)$ are two points of $\tilde{\chi}(f)$, we show that $\tilde{j}$ is generic by showing that $\left\{\tilde{j}_{*} \tilde{\chi}_{\left(x, y_{1}\right)}\right\}^{\perp} \subset \tilde{j}_{*} \tilde{\chi}_{\left(x, y_{2}\right)}$ (see the remark after 1.6). For, applying $f_{*}$ to the left side, we get:

$$
f_{*}\left\{\tilde{j}_{*} \tilde{\chi}_{\left(x, y_{1}\right)}\right\}^{\perp}=\left(f_{*} V_{x} \cap f_{*} V_{y_{1}}\right)^{\perp} \cap f_{*} V_{x}=f_{*} V_{y_{1}}^{\perp} \cap f_{*} V_{x}=f_{*} V_{y_{1}}^{\perp}
$$

by 1.6(a). Also applying $f_{*}$ to the right side we get $f_{*}\left\{\tilde{j}_{*} \tilde{\chi}_{\left(x, y_{2}\right)}\right\}=f_{*} V_{x} \cap f_{*} V_{y_{2}}$. But $f_{*} V_{y_{1}}^{\perp} \subset f_{*} V_{x} \cap f_{*} V_{y_{2}}$ by $1.6\left(\right.$ a) since $f(x)=f\left(y_{1}\right)=f\left(y_{2}\right)$. Hence $\left\{\tilde{J}_{*} \tilde{\chi}_{\left(x, y_{1}\right)}\right\}^{\perp} \subset \tilde{J}_{*} \tilde{\chi}_{\left(x, y_{2}\right)}$ as claimed.

1.8. Corollary. If $f: V \rightarrow M$ is a generic immersion between $k$-parallelizable manifolds, then $\tilde{\chi}(f)$ is $k$-parallelizable.

Proof. Since $\sigma^{*} \sigma^{*}=1$, we see $\nu(\tilde{j})=(\tilde{j} \sigma)^{*} \nu(f)$, and $\nu(f)$ is stably trivial on the $k$-skeleton. Hence $\tilde{j}$ is an immersion into a $k$-parallelizable manifold, with $\nu(\tilde{j})$ stably trivial on the $k$-skeleton. Thus $\tilde{\chi}(f)$ is $k$-parallelizable.

Finally we cite three results as lemmas, two are standard, and the third is trivial:

1.9. Lemma. Let $f: V^{n} \rightarrow M^{m}$ be any immersion between manifolds, and let $\iota: M-f(V) \rightarrow M$ be inclusion. Then $\iota_{*}: \pi_{k}(M-f(V)) \rightarrow \pi_{k}(M)$ is an epimorphism if $k<m-n$ and an isomorphism if $k<m-n-1$.

Proof. Obviously, by general position, any smooth map of $S^{k}$ (if $k<m-n$ ) or of $D^{k+1}$ (if $k<m-n-1$ ) into $M^{m}$ can be approximated by one whose image is disjoint from any given countable union of embedded $n$-manifolds (without changing that map on $\partial D^{k+1}$ if necessary). Since $f(V)$ can be written as such a union, this proves the theorem.

1.10. Lemma (ENGUlfing TheOREM). Let $j: X^{t} \rightarrow V^{n}$ be a generic immersion of manifolds, where $V$ is $t-q$ connected, $X$ is compact, and $H^{i}(X)=0$ (for all coefficient groups) for $t>i>t-q$, where $n-t-1>q>0$. Assume also that the single obstruction 
to deforming $j$ to a point, in $H^{t}\left(X ; \pi_{t}(V)\right)$, is zero. Then there is an $n$-disc $D^{n}$ in $V^{n}$ with $j(X) \subset D^{n}$.

Proof. Let $i: j(X) \rightarrow V^{n}$ be inclusion. We shall show that $i$ is null homotopic. If this is granted momentarily, it follows immediately from the main result of [8] that there is an $n$-disc $D^{n}$ in $V$ with $j(X) \subset D^{n}$. (Note: According to [8], this disc will be a piecewise linear disc, but its interior is then a smooth copy of $R^{n}$ in which we can choose a smooth ball of large enough radius to give the desired result.)

So we must show $i$ is null homotopic. First note that the double point set of $j$, denoted $Y \subset X$, is an immersed manifold of dimension $2 t-n<t-q$, and $j: X-Y$ $\approx j(X)-j(Y)$. So by excision, if $i>t-q \geqq \operatorname{dim}(Y)$, we have

$$
H^{i}(j(X)) \approx H^{i}(j(X), j(Y)) \approx H^{i}(X, Y) \approx H^{i}(X),
$$

and this last group is 0 for $t>i>t-q$. Hence the sole obstruction to a null homotopy of $i$ is in $H^{t}\left(X ; \pi_{t}(V)\right)$ and it coincides with the obstruction to the null homotopy of $j$, which is zero. This completes the proof.

1.11. LeMmA. If $f: V^{n} \rightarrow M^{m}$ is generic and $N$ is any manifold, then

$$
f \times 1: V^{n} \times N \rightarrow M^{m} \times N
$$

is generic, $\tilde{\chi}(f \times 1)=\tilde{\chi}(f) \times N$, and $\tilde{j}(f \times 1)=\tilde{j}(f) \times 1$.

The proof is obvious.

We remark that these results can be just as easily formulated and proved for generic immersions of manifolds with boundary. By a generic immersion of these we mean an immersion $f:(V, \partial V) \rightarrow(M, \partial M)$ such that $f($ Int $V) \subset$ Int $M, f \mid$ Int $V$ is generic, $f \mid \partial V: \partial V \rightarrow \partial M$ is generic, and in a collar neighborhood of $\partial V$ and $\partial M$, say $\partial V \times I$ and $\partial M \times I, f$ can be given by $(f \mid \partial V) \times 1$.

II. The double point set as a generalized homology class. In this section we introduce an invariant which, in favorable circumstances, will be the sole obstruction to changing an immersion into an embedding, through a homotopy. We refer the reader to [18] and [4] for information of generalized homology theories.

Throughout this chapter, $h_{*}$ will denote a generalized homology theory defined by a ring spectrum, with $h^{*}$ its dual cohomology theory. Thus $h_{*}$ and $h^{*}$ are multiplicative theories. Also, $h_{*}$ and $h^{*}$ are defined initially only for $\mathrm{CW}$ complexes with base point, $\left(X, x_{0}\right)$. As usual, they are extended to the category of CW pairs by the rule $h(X, A)=h(X / A, *)$ where $*$ is the obvious base point. Recall $X / \varnothing$ means $(X \cup *, *)$. We gather here, for the reader's convenience, some of the basic facts about $h$-orientable manifolds and bundles (see [18] or [4] for definitions).

2.1. Let $z$ be a fundamental class in $h_{n}(M, \partial M)$ for a compact $h$-oriented manifold with boundary. Let $N_{1}, N_{2} \subset \partial M$ be manifolds with $N_{1} \cap N_{2}=\partial N_{1} \cap \partial N_{2}$ and $N_{1} \cup N_{2}=\partial M$. Then $\bigcap z: h^{*}\left(M, N_{1}\right) \rightarrow h_{*}\left(M, N_{2}\right)$ is an isomorphism. (See [18] for the case where $\partial N_{1}=\partial N_{2}=\varnothing$. When $N_{1}=\partial M, N_{2}=\varnothing$, this then follows using 
the Mayer-Vietoris sequence for the double of $M$. The general case is then a consequence of the exact sequence of the triple $\left(M, \partial M, N_{1}\right)$.)

2.2. We can usually introduce Poincaré duality in noncompact manifolds as follows. For any space $X$, we define its cohomology with compact supports as $h_{c}^{*}(X)=\operatorname{inj} \operatorname{Lim} h^{*}(X, X-K)$ as $K$ ranges over the system of compact subsets of $X$. A manifold $M^{m}$ is called $h$-oriented if there is given, for each compact $m$-submanifold $N$, a fundamental class in $h_{*}(N, \partial N)$ such that $j_{*}\left(\left[N^{\prime}\right]\right)=[N]$, if $N \subset N^{\prime}$, and $j_{*}: h_{*}\left(N^{\prime}, \partial N^{\prime}\right) \rightarrow h_{*}\left(N^{\prime}, N^{\prime}-\right.$ Int $\left.N\right) \approx h_{*}(N, \partial N)$. Now assume $h_{*}$ satisfies the property $h_{*}(X)=\operatorname{inj} \operatorname{Lim} h_{*}(K)$ as $K$ ranges over the compact sets of $X$. It follows that the system of isomorphisms $\bigcap[N]$ combine to define an isomorphism $P: h_{c}^{*}(M) \rightarrow h_{*}(M)$ which has all the expected properties.

2.3. The natural fundamental classes for $S^{k-1}$ and $D^{k}$, say $\iota_{k-1}$ and $d_{k}$, induce a Hurewicz homomorphism $\varphi: \pi_{k}(X, A) \rightarrow h_{k}(X, A)$, and thence a natural transformation of homology theories $\varphi: \Pi_{*} \rightarrow h_{*}$, where $\Pi_{*}$ denotes stable homotopy theory.

2.4. Let $\xi^{n}$ be a vector bundle over $X$, and $A$ a subcomplex of $X$. Let $U$ in $h^{n}(D(\xi), S(\xi))$ be an orientation class for $\xi$, over $h_{*}$ (as in [3]). Then

$$
\cup U: h^{q}(X, A) \rightarrow h^{q+n}(D(\xi), S(\xi) \cup D(\xi \mid A))
$$

is an isomorphism, and so is $\bigcap U: h_{q+n}(D(\xi), S(\xi) \cup D(\xi \mid A)) \rightarrow h_{q}(X, A)$. (See Dold [3].) Furthermore if $\xi^{n}$ is a bundle over a compact manifold with boundary $M$, then $D(\xi)$ is a compact manifold with boundary, and an $h$-orientation (or fundamental class) for any two of these three objects $M, \xi, D(\xi)$, say $z, U, Z$, uniquely determines an $h$-orientation for the third by the equation $U \cap Z=z$. (The existence and uniqueness of such a $U, Z$ or $z$, given the other two, is clear from the Thom isomorphism or Poincaré duality on $D(\xi)$; that the class so determined is actually an orientation class is then a trivial verification.)

As a consequence of this, if $M$ and $N$ are $h$-oriented compact manifolds with boundary, and $(M, \partial M) \subset(N, \partial N)$, we see that $D(\nu) \subset N$ has an induced $h$-orientation from that of $N$, and this determines a unique orientation $U$ of $\nu$ by

$$
U \cap[D(v)]=[M] .
$$

2.5. Now suppose $i:(M, \partial M) \rightarrow(N, \partial N)$ is an immersion of compact manifolds, and $N$ is $h$-oriented. Let $j:(N, \partial N) \rightarrow(N, \partial N) \times D^{k}$ be inclusion, $j(x)=(x, 0)$, and let $i_{0}=j i$. If $[N]$ denotes the fundamental class for $N$, then $[N] \times d_{k}$ in

$$
h_{*}\left((N, \partial N) \times\left(D^{k}, S^{k-1}\right)\right)
$$

is a fundamental class for $N \times D^{k}$. If $k$ is sufficiently big, the map $i_{0}$ is regularly homotopic to an embedding $i_{1}$ (and any two such regular homotopies are themselves regularly homotopic). Hence this gives a uniquely determined bundle equivalence $\chi: \nu\left(i_{1}\right) \approx \nu\left(i_{0}\right)=\nu(i)+\varepsilon^{k}$. Hence if $Z^{\prime}$ is a fundamental class for $D\left(\nu\left(i_{1}\right)\right)$, then $\chi_{*}\left(Z^{\prime}\right)=Z^{\prime \prime}$ is a class of the form $Z \times d_{k}$ by suspension-here $(X, A) \times(Y, B)$ 
$=(X \times Y, X \times B \cup A \times Y) . Z$ is clearly a fundamental class for $D \nu(i)$ (e.g. use 2.4 where $\xi$ is trivial). Thus for any immersion $i:(M, \partial M) \rightarrow(N, \partial N)$ an $h$-orientation of $N$ uniquely determines an $h$-orientation $Z$ for the manifold $D \nu(i)$. It is an easy exercise to show that this orientation does not depend on $k$, and in fact, if $i$ is an embedding, this reduces to the usual orientation if we set $k=0$.

Hence if $i:(M, \partial M) \rightarrow(M, \partial M)$ is an immersion of $h$-oriented manifolds, by the preceding paragraph and 2.4 , there is a uniquely determined orientation $U_{i}$ of $\nu(i)$ satisfying $U_{i} \cap Z=[M], Z$ being the orientation of the manifold $D \nu(i)$ induced by the orientation of $N$.

2.6. Now let $V_{1}, V_{2}, M$ be $h$-oriented manifolds, $V_{i}$ compact, and let

$$
f_{i}:\left(V_{i}, \partial V_{i}\right) \rightarrow(M, \partial M)
$$

be generic immersions intersecting generically, $i=1,2$. Let $\chi\left(f_{1}, f_{2}\right), k, k^{\prime}$ be as in 1.5 with $\lambda: k^{*} \nu\left(f_{2}\right) \approx \nu(k)$ as in $1.7(\mathrm{~B})$. Then if $U_{2}$ is the orientation of $\nu\left(f_{2}\right)$ given by $2.5, \lambda^{*} k^{*} U_{2}$ is an orientation $U_{k}$ for $\nu(k)$. If $\left[D_{k}\right]$ is the fundamental class of $D v(k)$ given by 2.5 from $k: \chi \rightarrow V_{1}$, then $U_{k} \cap\left[D_{k}\right]$ gives a fundamental class $[\chi]$ for $\chi\left(f_{1}, f_{2}\right)$. This class depends only on the orientations of $V_{1}, M$ and $V_{2}$.

Now let $\zeta$ be the Poincaré dual of $f_{2 *}\left[V_{2}\right]$ in $h^{*}(M)$, and $\zeta^{\prime}$ the Poincaré dual of $k_{*}[\chi]$ in $h^{*}\left(V_{1}\right)$.

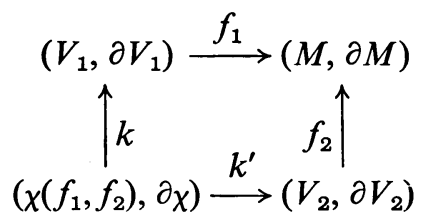

2.7. Lemma. $\zeta^{\prime}=f_{1}^{*}(\zeta)$.

Proof. (A) Assume first that $f_{2}$ is an imbedding. Then $k$ is as well, so $D \nu(k) \subset V_{1}$, $D \nu\left(f_{2}\right) \subset M, U_{2}$ is the Poincaré dual of $\left[V_{2}\right]$ on $D \nu\left(f_{2}\right), U_{k}$ is the Poincaré dual of [ $\chi$ ] on $D v(k)$ and $\lambda^{*} k^{*} U_{2}=U_{k}$ by naturality. Taking collapsing maps,

$$
c: M \rightarrow D \nu\left(f_{2}\right) / \partial D \nu\left(f_{2}\right) \text { and } c^{\prime}: V_{1} \rightarrow D \nu(k) / \partial D \nu(k),
$$

we see $\left[D_{f_{2}}\right]=c_{*}[M],\left[D_{k}\right]=c_{*}^{\prime}\left[V_{1}\right]$ by definition, and $\lambda^{*} k^{*} c^{*}=f_{1}^{*} c^{*}$, and so $\zeta^{\prime}=c^{*} U_{k}=c^{\prime *} \lambda^{*} k^{\prime *} U_{2}=f_{1}^{*} c^{*} U_{2}=f_{1}^{*} \zeta$ as claimed.

(B) Next assume $f_{2}$ extends to a generic immersion $\bar{f}_{2}:\left(V_{2}, \partial V_{2}\right) \times I \rightarrow(M, \partial M)$ intersecting $f_{1}$ generically, with $f_{2}(x)=\bar{f}_{2}(x, 0)$ and $\bar{f}_{2} \mid V_{2} \times 1$ an embedding. Then as in 2.6, $\chi\left(f_{1}, \bar{f}_{2}\right)$ has an $h$-orientation induced by those of $V_{1}, M$, and $V_{2} \times I$. Clearly when included into $h_{*}\left(V_{1}, \partial V_{1}\right)$ it shows that $k_{*}[\chi]$ is homologous to $\bar{k}_{*}[\tilde{x}]$ where $[\tilde{\chi}]$ is the fundamental class of $\chi\left(f_{1}, f_{2} \mid V_{2} \times 1\right)$ and $\bar{k}$ is the restriction of $\bar{k}: \chi\left(f_{1}, \bar{f}_{2}\right) \rightarrow V_{1}$. But by (A) the dual of $\bar{k}[\tilde{\chi}]$ is $f_{1}^{*}\left(\zeta_{1}\right)$ where $\zeta_{1}$ is the dual of $\bar{f}_{2 *} i_{1 *}\left[V_{2}\right], i_{1}: V_{2} \rightarrow V_{2} \times I$ being $i_{1}(x)=(x, 1)$. But $\bar{f}_{2} i_{1}$ is homotopic to $f_{2}$ so $\bar{f}_{2 *} i_{1 *}\left[V_{2}\right]=f_{2 *}\left[V_{2}\right]$ and $\zeta_{1}=\zeta$. Hence $f_{1}^{*} \zeta=\zeta^{\prime}$ in this case.

(C) Now suppose we cross the above diagram with $D^{k}$. We get $U_{f_{2} \times 1}=U_{2} \times \delta^{k}$, 
$\left[V_{2} \times D^{k}\right]=\left[V_{2}\right] \times d_{k}$, etc. (see 1.11) and finally we get $\zeta_{x \times D^{k}}^{\prime}=\zeta_{x}^{\prime} \times \delta^{k}, \quad \zeta_{V_{2} \times D^{k}}$ $=\zeta_{V_{2}} \times \delta^{k}$. So if the lemma is true here it is true in the general case. But if $k$ is big, $f_{2} \times 1$ satisfies condition (B), and so the lemma is proved.

2.8. Definition. Let $V^{n}$ and $M^{m}$ be $h$-oriented manifolds with boundary, $V$ compact, and let $f:(V, \partial V) \rightarrow(M, \partial M)$ be a continuous map. Define a class $\gamma(f)$ in $h_{r}(V, \partial V)$ by

$$
\gamma(f)=P_{V} f^{*} P_{M}^{-1} f_{*}[V], \quad r=2 n-m,
$$

where $P$ denotes Poincaré duality.

In the case where $V$ is given a base point $*$, we write $\tilde{\gamma}(f)$ for the image of $\gamma(f)$ in $h_{r}(V, \partial V \cup *)$. If $f^{-1} f(*)=*$, and we also write $*$ for $f(*)$, we see easily that $\tilde{\gamma}(f)$ is given by the composition

$$
\begin{aligned}
{[V] \in h_{n}(V, \partial V \cup *) \stackrel{f_{*}}{\longrightarrow} h_{n}(M, \partial M \cup *) \stackrel{P^{-1}}{\longrightarrow} h^{m-n}(M-*) } \\
\stackrel{f^{*}}{\longrightarrow} h^{m-n}(V-*) \stackrel{P}{\longrightarrow} h_{r}(V, \partial V \cup *) .
\end{aligned}
$$

2.9. Now let $f:(V, \partial V) \rightarrow(M, \partial M)$ be a generic immersion between $h$-oriented manifolds. Let $\tilde{j}: \tilde{\chi}(f) \rightarrow V$ be as in 1.5. It is a generic immersion. Let $v$ denote the normal bundle of $\tilde{j}$. By 1.7(A) there is an equivalence of bundles $\lambda: \nu \approx(j \sigma)^{*} \nu(f)$. Here $\sigma: \tilde{\chi}(f) \rightarrow \tilde{\chi}(f)$ is the covering transformation of 1.5. Since $V$ and $M$ are oriented, this defines an orientation class $U$ for $\nu(f)$ by 2.5. Hence $\lambda^{*}(\tilde{j} \sigma)^{*} U$ is an orientation $U_{j}$ for $\nu$. By 2.4 and 2.5, $U_{j}$ induces an orientation on $\tilde{\chi}(f)$ and we write the fundamental class as $[\tilde{x}(f)]$. It is thus determined by $f,[V]$ and $[M]$ alone. Note that $\tilde{j}_{*}[\tilde{x}(f)]$ is a class in $h_{r}(V, \partial V)$.

2.10. THEOREM. Let $f:\left(V^{n}, \partial V\right) \rightarrow\left(M^{m}, \partial M\right)$ be a generic immersion of h-oriented manifolds, $V$ compact, and assume $\nu(f)$ has a nowhere zero cross section. Then $\gamma(f)=j_{*}[\tilde{x}(f)]$. (Compare Smale-Lashof [12].)

Proof. We continue the notation of 2.9. Also for any bundle $\xi$ with metric, we write $\xi^{\varepsilon}, \varepsilon>0$, for its open $\varepsilon$-ball bundle, and $\xi^{0}$ for its zero section. Pick a Riemannian metric on $M$ satisfying 1.6 for $f: V \rightarrow M$, thus inducing a metric on $\nu(f), V$, $\tilde{\chi}(f)$ and $\nu$. Pick $\varepsilon>0$ so small that $\exp _{M} f_{*}: \nu^{\varepsilon}(f) \rightarrow M$ is an immersion, and pick a nowhere zero cross section $s: V \rightarrow \nu^{\varepsilon}(f)$ so close to the zero section (in the $C^{1}$ sense) that $f_{1}=\exp _{M} \circ f_{*} \circ s: V \rightarrow M$ is a generic immersion (cf. 1.2). Let $s_{1}: \tilde{\chi}(f) \rightarrow \nu^{\varepsilon}$ be induced by the equivalence of $1.7(\mathrm{~A})$ so that, for $z \in \tilde{\chi}(f)$,

$$
f_{*} \tilde{J}_{*} s_{1}(\sigma z)=f_{*} s \tilde{j}(z)
$$

Since $f(V)$ is totally geodesic,

$$
\exp _{M} f_{*}=f \exp _{V}: V_{x} \rightarrow M \text { for } x \in V .
$$

By (1), (2), and 1.7(A), if $z \in \tilde{\chi}(f)$,

$$
\exp _{M} f_{*} \nu^{\varepsilon}(f)_{\tilde{j}(z)}=\exp _{M}(f \tilde{j})_{*} \nu_{\sigma z}^{\varepsilon}=f \exp _{V} \tilde{J}_{*} \nu_{\sigma z}^{\varepsilon}
$$


Also if $x \in V-\tilde{X}(f), \exp _{M} f_{*} \nu^{\varepsilon}(f)_{x} \cap f(V)=f(x)$ if $\varepsilon$ is small. So considering $\exp _{M} f_{*}: \nu^{\varepsilon}(f) \rightarrow M$ we get

$$
\left(\exp _{M} f_{*}\right)^{-1} f(V)=\nu^{0}(f) \cup \nu^{\varepsilon}(f) \mid \tilde{X}(f) .
$$

So $f_{1}^{-1} f(V)=s_{1}^{-1} \nu^{\varepsilon}(f) \mid X(f)=\tilde{X}(f)$. Also $f_{1}$ intersects generically with $f$ since $s$ intersects generically with $\left(\exp _{M} f_{*}\right)^{-1} f(V)$-i.e. with $\nu^{\varepsilon}(f) \mid \tilde{X}(f)$ (as does any cross section). So $\tilde{j}(\tilde{\chi}(f))=k\left(\chi\left(f_{1}, f\right)\right)=f_{1}^{-1} f(V)$. By 1.3 there is a unique smooth map $h: \tilde{\chi}(f) \rightarrow \chi\left(f_{1}, f\right)$ with $k h=\tilde{j}$, and $h$ is a diffeomorphism. By (1) and (2) we see that one such map is $h(z)=\left(j(z), \exp _{V} j_{*} s_{1} \sigma z\right) \in \chi\left(f_{1}, f\right)$.

As in 2.9, $U_{j}=(\tilde{j} \sigma \lambda)^{*} U, \lambda:(\tilde{j} \sigma)^{*} \nu(f) \approx \nu(\tilde{j})$ as in 1.7(A). Also $\nu(k)$ over $\chi\left(f_{1}, f\right)$ has orientation class $U_{k}=\left(k^{\prime} \lambda^{\prime}\right)^{*} U,\left(\lambda^{\prime}: v(k) \approx k^{\prime *} v(f)\right.$ as in 1.7(B)). Now $h$ lifts to a bundle map $h: \nu(j) \rightarrow \nu(k)$, and one easily checks that $h \lambda=\lambda^{\prime} h:(j \sigma)^{*} \nu(f) \rightarrow \nu(k)$. Also, since $k^{\prime}(x, y)=y, k^{\prime} h(z)=\exp _{v} \tilde{j}_{*} s_{1}(\sigma z)$, and so the homotopy,

$$
k_{t}(z)=\exp _{v} \tilde{j}_{*}\left(t \cdot s_{1} \sigma z\right)
$$

is a regular homotopy between $k^{\prime} h$ and $\tilde{j} \sigma: \tilde{\chi}(f) \rightarrow V$. Thus we have

$$
h^{*} U_{k}=h^{*}\left(\lambda^{\prime *} k^{*} U\right)=\lambda^{*} h^{*} k^{*} U=\lambda^{*}(\tilde{j} \sigma)^{*} U=U_{\tilde{j}}
$$

So passing to homology, $h_{*}[\tilde{\chi}(f)]=\left[\chi\left(f_{1}, f\right)\right]$. By 2.7, then, $P_{V}^{-1} k_{*}\left[\chi\left(f_{1}, f\right)\right]=$ $f_{1}^{*} P_{M}^{-1} f_{*}[V]$. Since $f_{1}^{*}=f^{*}, j_{*}[\tilde{\chi}(f)]=k_{*}\left[\chi\left(f_{1}, f\right)\right]=P_{V} f^{*} P_{M}^{-1} f_{*}[V]=\gamma(f)$ as claimed.

Our interest in 2.10 will be when $h^{*}$ is some cobordism theory. We now wish to describe these cobordism theories.

2.11. Let $k>0$ be a fixed integer, $X$ an object in the category of pointed spaces having the homotopy type of a $\mathrm{CW}$ complex and homotopy classes of maps of these (preserving base points). Let the map $\pi(k):{ }_{k} X \rightarrow X$ denote the $k$-connective fiber space over $X$. Thus ${ }_{k} X$ is $k$-connected, $\pi(k)$ is a fiber map and $\pi(k)_{*}: \pi_{q}\left({ }_{k} X\right) \rightarrow \pi_{q}(X)$ is an isomorphism for all $q>k$. It is easily seen that the correspondence $X \rightarrow{ }_{k} X$ is a functor on the above mentioned category into itself, and we write ${ }_{k} f:{ }_{k} X \rightarrow{ }_{k} Y$ for the map induced by $f: X \rightarrow Y$. It can be chosen as a map of fiber spaces.

Let $\gamma^{n}$ be the universal oriented $n$-plane bundle, with base space $B S O(n)$. We set ${ }^{k} \gamma^{n}=\pi(k)^{*}\left(\gamma^{n}\right)$, where $\pi(k):{ }_{k} B S O(n) \rightarrow B S O(n)$, and we denote the Thom space of the bundle ${ }^{k} \gamma^{n}$ by ${ }^{k} M S O(n)$. Let $j_{n}: B S O(n) \rightarrow B S O(n+1)$ be induced by $S O(n)$ $\subset S O(n+1)$. Since $\pi(k) \circ{ }_{k} j_{n}=j_{n} \pi(k)$, we see that for ${ }_{k} j_{n}:{ }_{k} B S O(n) \rightarrow{ }_{k} B S O(n+1)$, we have ${ }_{k} j_{n}^{*}\left({ }^{k} \gamma^{n+1}\right)={ }^{k} \gamma^{n} \oplus \varepsilon^{1}$. Thus the diagram below commutes up to homotopy, where $S$ is the suspension functor and $M(f)$ denotes the map of Thom spaces induced by a bundle map $f$.

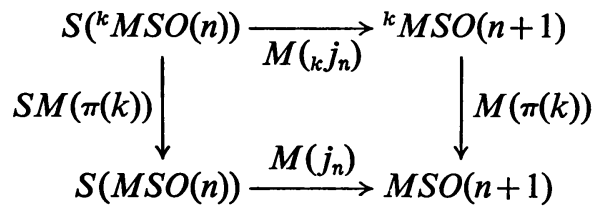


The maps $M\left({ }_{k} j_{n}\right)$ will be denoted ${ }^{k} j_{n}$; these combine to define a spectrum, denoted ${ }^{k} M S O$, and the maps $M(\pi(k))$ define a map of spectra,

$$
M \pi(k):{ }^{k} M S O \rightarrow M S O .
$$

If $\mu: B S O(m) \times B S O(n) \rightarrow B S O(m+n)$ is induced by $S O(m) \times S O(n) \rightarrow S O(m+n)$, this lifts to a map $\mu:{ }^{k} M S O(m) \times{ }^{k} M S O(n) \rightarrow{ }^{k} M S O(n+n)$. This gives a pairing $\mu:\left({ }^{k} M S O,{ }^{k} M S O\right) \rightarrow{ }^{k} M S O$, clearly making ${ }^{k} M S O$ into a ring spectrum. It is also clear that $M \pi(k):{ }^{k} M S O \rightarrow M S O$ is a map of ring spectra, as is the map $i: S \rightarrow{ }^{k} M S O$ ( $S$ is the sphere spectrum) obtained by considering the image of the fiber of ${ }^{k} \gamma^{n}$ over * as an oriented $n$-sphere in ${ }^{k} M S O(n)$. Finally the natural map, for each $X$, of ${ }_{k+1} X$ into ${ }_{k} X$ lifts to a map of ring spectra $\pi:{ }^{k+1} M S O \rightarrow{ }^{k} M S O$.

Each of these spectra ${ }^{k} M S O$ define homology and cohomology theories on the category of $\mathrm{CW}$ complexes, as in [18]. We now sketch briefly an explicit cobordism interpretation of these theories.

2.12. For any smooth manifold $M$ with stable normal bundle $\nu^{N}$, we say that a $k$-framing is a trivialization of $\nu$ over the $k-1$-skeleton of $M$, which can be extended in some way to a trivialization on the $k$-skeleton. Two such $k$-framings are equivalent if the framings they give on $M \times 0$ and $M \times 1$ in $M \times I$ extend to a $k$-framing on all of $M \times I$. It is easily seen that a $k$-framing of $M$ relative to one $\mathrm{CW}$ decomposition induces a $k$-framing relative to any other decomposition by taking a cellular approximation to the identity map, and this $k$-framing is unique up to equivalence.

Now for any CW complex $X$, with $k$-skeleton $X^{k}$, and for any space $Y$, we write $[X, Y]_{k}$ for the image of $\left[\left(X, X^{k}\right),(Y, *)\right]$ in $\left[\left(X, X^{k-1}\right),(Y, *)\right]$. Thus an equivalence class of $k$-framings for $M$ is clearly an element of $[M, B S O]_{k}$ which maps to $v$ under the natural map $[M, B S O]_{k} \rightarrow[M, B S O]$.

2.13. Lemma. For $X, Y, X^{k},{ }_{k} Y$, as before, there is a natural bijection of sets $\left[X,{ }_{k} Y\right] \rightarrow[X, Y]_{k}$ induced by $\pi(k):{ }_{k} Y \rightarrow Y$.

Proof. It is clear that $\left[X,{ }_{k} Y\right]_{k} \rightarrow\left[X,{ }_{k} Y\right]$ is a bijection, ${ }_{k} Y$ being $k$-connected. So $\pi(k)_{*}$ does indeed map $\left[X,{ }_{k} Y\right]$ into $[X, Y]_{k}$ in the obvious way. Now let $f$ represent an element of $[X, Y]_{k}$. Since $f\left(X^{k-1}\right)=*$, and $f \mid X^{k}$ is null homotopic rel $X^{k-1}$, there is a map $g: X^{k} \rightarrow{ }_{k} Y$, with $\pi(k) g=f$, and $g\left(X^{k-1}\right)=*$. Also $g$ is null homotopic rel $X^{k-1}$ (since ${ }_{k} Y$ is $k$-connected), so any two such liftings are certainly homotopic rel $X^{k-1}$. Now $g$ extends to a lifting of $f$ over all of $X$, and two such liftings are homotopic rel $X^{k-1}$, the obstructions lying in the groups $\pi_{q}(F)$ and $\pi_{q+1}(F)$ respectively, $q \geqq k$, which are zero $\left(F\right.$ is the fiber of ${ }_{k} Y \rightarrow Y$ ). Hence the correspondence $\left[X,{ }_{k} Y\right]_{k} \rightarrow[X, Y]_{k}$ is a bijection and we are done.

Thus, by the lemma, an equivalence class of $k$-framings of $M$ corresponds to an element of $\left[M,{ }_{k} B S O\right]$ which maps to $\nu$ under ${ }_{k} B S O \rightarrow B S O$.

Let us say that a $k$-framed manifold is a manifold $M^{n}$ with an equivalence class of $k$-framings. We say $M$ bounds if $M$ is closed, and there is a compact $k$-framed 
manifold $W^{n+1}$ such that $M=\partial W$, and the $k$-framing of $W$ restricts to that of $M$ (i.e. by adding the interior normal vector along the boundary as the last vector of the $k$-framing of $M$ ). The negative of a given $k$-framed manifold is understood to be the same manifold and framing, except that the last vector of the framing is reversed. Two framed manifolds are cobordant if their difference bounds; this is an equivalence relation, and the equivalence class yield a group, ${ }^{k} \Omega_{n}$. A $k$-framing of $M$ gives a bundle map $E\left(\nu^{N}\right) \rightarrow E\left({ }^{k} \gamma^{N}\right)$ and thus, as usual (see Thom [14]) we get a map $S^{n+N} \rightarrow{ }^{k} M S O(N)$ which defines the usual isomorphism, ${ }^{k} \Omega_{n} \approx \Pi_{n}\left({ }^{k} M S O\right)$. More generally, for any CW pair $(X, A)$, we can define ${ }^{k} \Omega_{n}(X, A)$ by taking cobordism classes of pairs $(M, f)$ where $M$ is a compact $k$-framed manifold, and $f:(M, \partial M) \rightarrow(X, A)$. By standard arguments we see that

$$
{ }^{k} \Omega_{*}(X, A) \approx \Pi_{*}\left({ }^{k} M S O \wedge(X / A), *\right),
$$

the homology theory determined by the spectrum ${ }^{k} M S O$. Henceforward, we write ${ }^{k} \Omega_{*}$ and ${ }^{k} \Omega^{*}$ for the homology and cohomology theory given by ${ }^{k} M S O$.

We need one more observation to complete this geometrical interpretation of ${ }^{k} \Omega_{*}$. Let $M^{n}$ be a compact $k$-framed manifold. Thus $(M$, id $)$ determines an element of ${ }^{k} \Omega_{n}(M)$, which we denote $[M]$.

2.14. Lemma. [M] is a fundamental class for $M$.

Proof. We must show that if $\zeta$ in ${ }^{k} \Omega^{n}(M)$ is the class given by the map

$$
M \stackrel{f}{\longrightarrow} S^{n} \stackrel{i}{\longrightarrow}{ }^{k} M S O(n)
$$

where $f: M^{n} \rightarrow S^{n}$ has degree one, then $\zeta /[M]$ in ${ }^{k} \Omega_{0}$ (point) $=\Pi_{0}\left({ }^{k} M S O\right)$ is the generator. But $\zeta /[M]$ is the composition

$$
\begin{aligned}
S^{N+n} \longrightarrow M\left(\nu^{N}\right) & \stackrel{\text { id } \wedge \pi}{\longrightarrow} M\left(\nu^{N}\right) \wedge(M / \varnothing) \longrightarrow{ }^{k} M S O(N) \wedge M / \varnothing \\
& \stackrel{\text { id } \wedge f}{\longrightarrow}{ }^{k} M S O(N) \wedge S^{n} \longrightarrow{ }^{k} M S O(N+n) .
\end{aligned}
$$

It is an easy exercise in (ordinary) cohomology theory and Poincaré duality to compute that this map, say $\alpha$, induces an isomorphism

$$
\alpha^{*}: H^{N+n}\left({ }^{k} M S O(N+n)\right) \rightarrow H^{N+n}\left(S^{N+n}\right) .
$$

From Hurewicz's theorem, and the universal coefficient theorem, it then follows that $\zeta /[M]$ is the generator in $\Pi_{N+n}\left({ }^{k} M S O(N+n)\right)$ as claimed.

To close this section, we prove a simple lemma about ${ }^{k} \Omega_{n}$ which will however be of great importance to us later.

2.15. Lemma. Let $X$ be a $k$-connected space with base point, $k \geqq 0$. Then for all $q<2 k$, the Hurewicz map $\varphi: \pi_{q}\left(X, x_{0}\right) \rightarrow{ }^{k} \Omega_{q}\left(X, x_{0}\right)$ is an isomorphism.

Proof. The map $i: S^{N} \rightarrow{ }^{k} M S O(N)$ is an embedding, and it is an immediate consequence of the Thom isomorphism that the pair $\left({ }^{k} M S O(N), S^{N}\right)$ is $N+k$ 
connected. Since $\left(X, x_{0}\right)$ is $k$-connected, the product of these, $\left(X \wedge{ }^{k} M S O(N), X \wedge S^{N}\right)$ is $N+2 k$ connected by the Kunneth formula. Therefore the homomorphism $\pi_{q}\left(X \wedge S^{N}\right) \rightarrow \pi_{q}\left(X \wedge{ }^{k} M S O(N)\right)$ is an isomorphism for $q<N+2 k$. This simply means that the Hurewicz map $\varphi: \Pi_{q}\left(X, x_{0}\right) \rightarrow{ }^{k} \Omega_{q}\left(X, x_{0}\right)$ is an isomorphism for $q<2 k$. By stability [1], $\Pi_{q}\left(X, x_{0}\right)=\pi_{q}\left(X, x_{0}\right)$ which proves the lemma.

A proof of 2.15 along the lines of Kervaire-Milnor [11] would also be possible.

\section{Surgery of self intersections and the main results.}

A. Surgery. In this section we exhibit the last tool necessary to establish our results: that of surgery on the self intersection set $X(f)$ of an immersion. The main idea here is that in favorable cases, we can modify $X(f)$ by surgery simply by changing $f$ by a regular homotopy (Proposition 3.3). This is, in fact, a simple modification of a special case of a construction of Haefliger (see [5, §3]). This method also appears, in essence, in [17] but the dimensional and connectivity restrictions imposed there are about twice as strict there as we want them here.

3.1. We quickly recall a construction due, in more general form, to Haefliger [5] whose notation we preserve. The reader should consult $[5, \S 3]$ for a fuller account of these ideas.

For a fixed $p>0$, let $D=D_{0}^{p} \cup D_{1}^{p}$ (disjoint) where each $D_{q}^{p}$ is a copy of the open $p$-disc of radius $\sqrt{ } 2$. Let $J$ be the involution which interchanges the two discs. Also $I=[-1,1]$ has an involution $J(t)=-t$. Let $D^{\prime}=D \times_{J} I$. Let $\lambda$ be the Morse function on $D$ such that $\lambda J=\lambda$ and $\lambda(d)=1-|d|^{2}$. Note $-1 \leqq \lambda \leqq 1$. The map $\varphi: D \rightarrow D^{\prime}$, given by $\varphi(d)=[d, \lambda(d)]$, is a generic immersion whose double point set is $D_{0}=\{d \in D: \lambda(d)=0\}[5, \S 3]$. Denote the total space of the trivial bundle of dimension $m-n-1$ on $D$ by $L$, and write $L \oplus_{J} L$ for $L \oplus J^{*} L$. Then $J: D \rightarrow D$ is covered by a bundle map, $J_{*}: L \oplus_{J} L \rightarrow L \oplus_{J} L$, given by $J_{*}\left(l_{d}, l_{J d}\right)=\left(-l_{J d},-l_{d}\right)$, and so we can for a bundle $L^{\prime}=\left(L \oplus_{J} L\right) \times{ }_{J} I$ over $D^{\prime}$. Then $\varphi: D \rightarrow D^{\prime}$ extends to a generic immersion $\Phi: L \rightarrow L^{\prime}$ (which is also a bundle monomorphism over $\varphi$ ) given by $\Phi\left(l_{d}\right)=\left[l_{d}, 0, \lambda(d)\right]$. Its double point set is still $D_{0}$, and we write $D_{0}^{\prime}$ for $\Phi\left(D_{0}\right)[5, \S 3]$. Note $D_{0}$ is just the two copies of the unit $p-1$ sphere, and $D_{0}^{\prime}$ is a single $p-1$ sphere.

There is a regular homotopy $\Phi_{t}: L \rightarrow L^{\prime}$ with $\Phi_{0}=\Phi$, such that $\Phi_{1}$ is an embedding. Specifically,

$$
\Phi_{t}\left(l_{d}\right)=\left[l_{d}, 0, \lambda(d)-\alpha\left(\left|l_{d}\right|\right) \cdot t \mu(d)\right] \quad(\mathrm{cf} .[5, \S 3.2])
$$

where $\mu(d)=\beta(|d|)(\lambda(d)+1)$, and $\alpha, \beta$ are $C^{\infty}$ functions such that $\alpha(t)=1$ if $t \leqq 0$, $\alpha(t)=0$ if $t \geqq 1, \alpha^{\prime}(t)<0$ if $0<t<1, \beta(t)=1$ if $t \leqq 1, \beta(t)=0$ if $t \geqq(3 / 2)^{1 / 2}, \beta^{\prime}(t)<0$ if $1<t<(3 / 2)^{1 / 2}$. Note that then $\mu(d)>\lambda(d)$ and $\lambda(d) / \mu(d)$ is a monotone function of $|d|$ for $d<(3 / 2)^{1 / 2}$.

Now suppose for 3.2 through 3.4 that $f: V^{n} \rightarrow M^{m}$ is a generic immersion without triple points. Hence $\chi(f)=X(f)$.

3.2. Definition. An $H$-construction for an embedded $p-1$ sphere $\iota: S^{p-1} \rightarrow X(f)$ shall mean: 
(a) Two embeddings $\psi: D \rightarrow V, \psi^{\prime}: D^{\prime} \rightarrow M$ such that $f \psi=\psi^{\prime} \varphi$, and $\psi^{-1}(\tilde{X}(f))$ $=D_{0} ; \psi^{\prime-1}(f(V))=\varphi(D)$ with the intersection of $D$ with $\tilde{X}(f)$, and $D^{\prime}$ with $f(V)$ both transverse, and $\psi^{\prime}\left(D_{0}^{\prime}\right)=\iota\left(S^{p-1}\right)$.

(b) Two bundle monomorphisms $\Psi_{*}: L \rightarrow \nu(\psi(D)), \Psi_{*}^{\prime}: L^{\prime} \rightarrow \nu\left(\psi^{\prime}\left(D^{\prime}\right)\right)$ covering $\psi$ and $\psi^{\prime}$ such that $f_{*} \Psi_{*}=\Psi_{*}^{\prime} \Phi ; \Psi_{*}^{-1} \nu(X(f))=\left.L\right|_{D_{0}} ; \Psi_{*}^{\prime \prime-1}\left(f_{*} \tau V\right)=\Phi(L)$. $(\nu$ and $\tau$ denote normal and tangent bundles.)

3.3. Proposition. If $\imath: S^{p-1} \rightarrow X(f)$ has an $H$-construction, there is a regular homotopy $f_{t}: V \rightarrow M$ with $f_{0}=f$ such that $f_{1}$ is generic and $X\left(f_{1}\right)$ is the manifold obtained from $X(f)$ by performing surgery on $\left(\left(S^{p-1}\right)\right.$ using some framing.

Proof. Using the metric of 1.3, the exponential map gives extensions of $\psi$ and $\psi^{\prime}$ to embeddings $\Psi: L_{\varepsilon} \rightarrow V, \Psi^{\prime \prime}: L_{\varepsilon}^{\prime} \rightarrow M$ with $f \Psi=\Psi^{\prime \prime} \Phi, \Psi^{\prime-1}(f(V))=\Phi\left(L_{\varepsilon}\right)$ (compare [5, §4.3]). Note that $\psi^{\prime}\left(D_{0}^{\prime}\right)$ in $X(f), \Psi^{\prime \prime}\left(L^{\prime}\right)$ in $M$, and $\Psi(L)$ in $V$ all have codimension $r-p+1\left(r=2 n-m\right.$, as always). Since $L^{\prime}$ is contractible there is a framing of $L^{\prime}$ in $M$ which induces a framing of $L$ in $V$ and a framing of $\psi^{\prime}\left(D_{0}^{\prime}\right)$ $=\iota\left(S^{p-1}\right)$ in $X(f)$ (this framing will give the surgery). These framings, together with the exponential map of the metric of 1.3, give embeddings $T: L_{\varepsilon} \times R^{r-p+1} \rightarrow V$, $T^{\prime}: L_{\varepsilon}^{\prime} \times R^{r-p+1} \rightarrow M$ with $f \circ T(l, v)=T^{\prime}(\Phi(l), v)$ and the images are open in $V$ and $M$. Define a regular homotopy $F_{t}: L \times R^{r-p+1} \rightarrow L^{\prime} \times R^{r-p+1}$ by $F_{t}(l, v)$ $=\left(\Phi_{t \alpha(|v|)}(l), v\right)$ where $\alpha$ is as in 3.1. So $F_{0}=\Phi \times$ identity. Hence its self intersection set is $D_{0}^{\prime} \times R^{r-p+1}$, which is diffeomorphic to $S^{p-1} \times R^{r-p+1}$ by the correspondence $([d, J d, 0], v) \mapsto(d, v)$. The self intersection set of $F_{1}$ is easily computed to be all $([d, J d, 0], v)$ such that $\lambda(d)-\alpha(|v|) \mu(d)=0$. The correspondence $([d, J d, 0], v)$ $\rightarrow(|v| d, v /|v|)$ thus gives a diffeomorphism of this self intersection set onto $R^{p} \times S^{r-p}$, showing that this set is obtained from the self intersection set of $F_{0}$ by surgery on the $p-1$ sphere $D_{0}^{\prime}$. It is trivial to check that $F_{1}$ is generic. Also note that $F_{t}$ and $F_{0}$ agree outside a compact set.

So to complete the proof, define $f_{t}: V \rightarrow M$ by $f_{t}=\Psi^{\prime \prime} F_{t} \Psi^{-1}$ on Image $\Psi, f_{t}=f$ elsewhere.

3.4. Proposition. Let $\iota: S^{p-1} \rightarrow X(f)$ be an embedding representing a class in $\pi_{p-1}(X(f))$ which is in the image of $\pi_{p-1}(\tilde{X}(f))$. Suppose $\pi_{p}(M)=\pi_{p-1}(V)=0$, and $0<p<m-n-1, p \leqq(r+1) / 2$. Then ، has an H-construction.

Proof. By covering space theory, ‘ lifts to two disjoint embeddings of $S^{p-1}$ in $\tilde{X}(f)$ and these bound disjoint discs in $V$, each intersecting $\tilde{X}(f)$ transversely and only on their boundaries. This is by general position, $p<m-n, 2 p<n$. Hence these give an embedding $\psi: D \rightarrow V$ with $\psi^{-1}(\tilde{X}(f))=D_{0}$. We can define $\psi^{\prime}: D^{\prime} \rightarrow M$ at least on $\varphi(D)$ by requiring $f \psi=\psi^{\prime} \varphi$. Now since $\varphi(D)$ is a $p$-sphere in homotopy type, the only obstruction to extending $\psi^{\prime}$ continuously over all of $D^{\prime}$ is in $\pi_{p}(M)=0$. So $\psi^{\prime}$ can be extended to a smooth embedding and by general position $(p+1<m / 2$, $p+1<m-n)$ we can assume $\psi^{\prime-1}(f(V))=\varphi(D)$ with $\psi^{\prime}\left(D^{\prime}\right)$ meeting $f(V)$ transversely. 
Now $\left.\nu \tilde{X}(f)\right|_{\psi\left(D_{0}\right)}$ is trivial by $1.7(\mathrm{~A})$, since $\left.\left.\nu(f)\right|_{\psi\left(D_{0}\right)} \subset \nu(f)\right|_{\psi(D)}$ must be trivial. This bundle has a section, consisting of vectors tangent to $\psi(D)$ but normal to $D_{0}$, and the orthogonal complement of this section is still trivial since it is stably trivial with dimension $m-n-1>p-1$. So this sub-bundle admits an $m-n-1$ frame which gives us an $m-n-1$ frame on $\left.\nu \psi(D)\right|_{\psi\left(D_{0}\right)}$. This extends to an $m-n-1$ frame on all of $\nu(\psi(D))$ since the obstruction is in $\pi_{p-1}\left(V_{n-p, m-n-1}\right)=0$ since $2 p \leqq r+1$. This $m-n-1$ frame gives a bundle map covering $\psi, \Psi_{*}: L \rightarrow \nu(\psi(D))$ and clearly $\Psi_{*}^{-1} \nu(\tilde{X}(f))=\left.L\right|_{D_{0}}$.

Now we define $\Psi_{*}^{\prime}: L^{\prime} \rightarrow \nu \psi^{\prime}\left(D^{\prime}\right)$. On $\Phi(L)$ we can define $\Psi_{*}^{\prime}$ by $f_{*} \Psi_{*}=\Psi_{*}^{\prime \prime} \Phi$. Thus on $\left.L^{\prime}\right|_{D_{0}^{\prime}} \Psi_{*}^{\prime}$ is defined on two complementary sub-bundles which are mapped monomorphically by $\Psi_{*}^{\prime}$ to two mutually orthogonal sub-bundles of $\left.\nu \tilde{X}(f)\right|_{\iota\left(S^{p-1}\right)}$. By linearity $\Psi_{*}^{\prime \prime}$ extends monomorphically over all of $\left.L^{\prime}\right|_{D_{0}^{\prime}}$. Now $\Psi_{*}^{\prime \prime}$ has been defined on a sub-bundle of $\left.L^{\prime}\right|_{\varphi\left(D_{q}^{p}\right)}$ having codimension $m-n-1$ for each $q$. In order to extend $\Psi_{*}^{\prime}$ monomorphically over each $\left.L^{\prime}\right|_{\varphi\left(D_{q}^{p}\right)}$ consistent with its values on $\left.L^{\prime}\right|_{D_{0}^{\prime}}$, we must extend an $m-n-1$ frame from each of the spheres of $D_{0}$ to the discs $D_{q}^{p}$. This frame must stay normal to the image of the above mentioned subbundle of $\left.L^{\prime}\right|_{D_{q}^{p}}$ (with dimension $m-n-1$ ), and must lie in $\nu\left(\psi^{\prime}\left(D^{\prime}\right)\right.$ ) (which has dimension $m-p+1)$. So the obstruction is in $\pi_{p-1}\left(V_{n-p, m-n-1}\right)=0$ as before. Thus $\Psi_{*}^{\prime \prime}$ is defined on $\left.L^{\prime}\right|_{\varphi(D)}$, and only $\Phi(L)$ maps into $f_{*}(\tau V)$. To extend $\Psi_{*}^{\prime \prime}$ over all of $D^{\prime}$ now amounts to extending a $2(m-n-1)$ frame in $\nu\left(\psi^{\prime}\left(D^{\prime}\right)\right)$ from a $p$-sphere-i.e. $\psi^{\prime}(\varphi(D))$-to a $p+1$ disc-namely $\psi^{\prime}\left(D^{\prime}\right)$. The obstruction, in $\pi_{p}\left(V_{m-p, 2(m-n-1)}\right)$ is zero since $2 p \leqq r+1$. So $\Psi_{*}^{\prime}$ is defined on all of $L^{\prime}$, with $f_{*} \Psi_{*}=\Psi_{*}^{\prime \prime} \Phi, \Psi_{*}^{\prime-1}\left(f_{*} \tau V\right)=\Phi(L)$. So the $H$-construction is complete.

The following corollary is what makes this method useful to us.

3.4.1. Corollary. Let $f: V^{n} \rightarrow M^{m}$ be any immersion, with $V$ compact and $[r / 2]-1$ connected, and $M[r / 2]$-connected. Assume $m \geqq(4 / 3)(n+1)$. Then $f$ is regularly homotopic to a generic immersion $f_{1}$ such that $\tilde{\chi}\left(f_{1}\right)$ is $([r / 2]-1)$-connected.

Proof. We may assume $f$ is generic by 1.2. We will try to perform surgeries on $X(f)$ to get the result.

We may as well assume $f$ has no triple points. Otherwise, if $\tilde{Y}(f)$ is its triple point set, consider $V^{\prime}=V-\tilde{Y}(f), M^{\prime}=M-f(\tilde{Y}(f)), f^{\prime}=f \mid V^{\prime}$. Then $f^{\prime}$ has no triple points, $\tilde{\chi}\left(f^{\prime}\right)=\tilde{\chi}(f)-\chi(\tilde{j})($ by $1.7(\mathrm{~A}))$ and since $\tilde{j}: \tilde{\chi}(f) \rightarrow V$ is generic (by $\left.1.7(\mathrm{~A})\right), \tilde{\chi}(\tilde{j})$ has codimension $m-n$ in $\tilde{\chi}(f)$. Thus $\pi_{p}\left(\tilde{\chi}\left(f^{\prime}\right)\right) \rightarrow \pi_{p}(\tilde{\chi}(f))$ is an isomorphism for $p \leqq[r / 2]-1$. (The inequality $m \geqq(4 / 3)(n+1)$ implies $[r / 2]<m-n-1$.) Similarly, $V^{\prime}$ and $M^{\prime}$ are just as connected as $V$ and $M$ by 1.9 , so if we modify $\tilde{X}\left(f^{\prime}\right)$ by a regular homotopy which is fixed off a compact set and makes $\tilde{X}\left(f^{\prime}\right)([r / 2]-1)$-connected, then we extend the homotopy over all of $V$ and this makes $\tilde{\chi}(f)$ just as connected.

Next we show that we may as well assume that $\tilde{\chi}(f)$ is connected: Since $f$ is generic and has no triple points, we can, using 3.3 and 3.4, make $\chi(f)$ connected by surgery on 0 -spheres, and hence we can at least assume that $\chi(f)$ is connected. Since $\pi: \tilde{\chi}(f) \rightarrow \chi(f)$ is a double covering, if $\tilde{\chi}(f)$ is not connected, it is just two 
copies of $\chi(f)$. In this case let $\iota: S^{0} \rightarrow \chi(f)$ be any embedding. Let $\iota_{0}, \iota_{1}$ be two liftings of $\iota$ to $S^{0} \rightarrow \tilde{\chi}(f)$ such that $\iota_{0}\left(S^{0}\right)$ and $\iota_{1}\left(S^{0}\right)$ both meet both the components of $\tilde{\chi}(f)$. As seen in the proof of 3.4 we can use these to give an $H$-construction for $\iota\left(S^{0}\right)$. In this $H$-construction, $\psi(D)$ consists of two intervals, each touching both components of $\tilde{X}(f)$, and each is the core disc of one of the two surgeries of $\tilde{X}(f)$ involved in the construction. Hence after applying 3.3 to this case $\tilde{X}(f)$ becomes connected as required.

Now, since $\tilde{\chi}(f)$ is connected, we begin performing surgery on $\chi(f)$ by killing all classes of $\pi_{p}(\chi(f))$ which are in the monomorphic image of $\pi_{p}(\tilde{\chi}(f))$ for $1 \leqq p$ $\leqq[r / 2]-1$. Since these are finitely generated, only a finite number of surgeries are required and the resulting homotopy is fixed off a compact set (as required in the second paragraph). In the end $\tilde{\chi}(f)$ is $([r / 2]-1)$-connected as required.

B. Proof of the main results. We can now proceed to the main theorem. In the sequel we will write $s$ for $[(r+1) / 2]$. Note that if a manifold $V$ is $s$-connected it admits an $s$-framing and so it is orientable over the theory $s \Omega$ of Chapter II. (Note there are exactly two such orientations in this case.)

3.5. THEOREM. Let $f: V^{n} \rightarrow M^{m}$ be an immersion whose normal bundle admits a cross section. Suppose $V$ is closed and s-connected, and $M$ is $(r+1)$-connected. Suppose $\tilde{\gamma}(f)=0$ over $^{s} \Omega$. Then:

(a) If $m \geqq(3 / 2)(n+1), f$ is homotopic to a smooth embedding.

(b) If $m \geqq(4 / 3)(n+1), n \neq 2, f$ is homotopic to a p.l. embedding which is a smooth embedding outside an $n$-disc of $V$.

Proof. By 3.4.1 we can assume $f$ is generic and $\tilde{\chi}(f)$ is $([r / 2]-1)$-connected. Let $D^{r}$ be a closed $r$-disc in $\tilde{\chi}(f)$, disjoint from the double point set of $\tilde{j}$. We wish to apply 1.10 to the map $\tilde{j}: \tilde{\chi}(f) \rightarrow V$. Note $V$ is $s$-connected and by Poincaré duality, $H^{i}(\tilde{\chi}(f))=0$ for $s<i<r$. So, by obstruction theory, $\tilde{j}$ is homotopic to a map $j^{\prime}: \tilde{\chi}(f) \rightarrow V$ such that $j^{\prime}\left(\tilde{\chi}(f)-D^{r}\right)$ is a point $v_{0}$ in $V$. The obstruction to making a null homotopy of $\tilde{j}$, which lies in $H^{r}\left(\tilde{\chi}(f) ; \pi_{r}\left(V, v_{0}\right)\right) \approx \pi_{r}\left(V, v_{0}\right)$ is now easily described. It is the class given by $j^{\prime}:\left(D^{r}, \partial D^{r}\right) \rightarrow\left(V, v_{0}\right)$, in $\pi_{r}\left(V, v_{0}\right)$. But by 2.15 , the Hurewicz map $\varphi: \pi_{r}\left(V, v_{0}\right) \rightarrow{ }^{s} \Omega_{r}\left(V, v_{0}\right)$ is injective, and it is clear that the image of this class is just $j_{*}^{\prime}[\tilde{x}(f)]$ in ${ }^{s} \Omega_{r}\left(V, v_{0}\right)$ which is just $\tilde{j}_{*}[\tilde{\chi}(f)]=\tilde{\gamma}(f)$ by 2.10 . Since $\tilde{\gamma}(f)=0$ by assumption, we can apply 1.10 and conclude that there is an embedded $n$-disc $D^{n}$ in $V^{n}$ with $\tilde{X}(f) \subset \operatorname{Int}\left(D^{n}\right)$.

Now let $V_{0}=f\left(V-D^{n}\right)$, and let $T$ be a tubular neighborhood of $V_{0}$ disjoint from $f\left(D^{n}\right)$ and chosen so that $M-T=M_{0}$ is a manifold with boundary. $M_{0}$ is then a deformation retract of $M-V_{0}$ and $f\left(\partial D^{n}\right)$ is contained in its boundary. Let $\psi=f \mid D^{n}:\left(D^{n}, \partial D^{n}\right) \rightarrow\left(M_{0}, \partial M_{0}\right)$. Note $\psi$ is an embedding in a neighborhood of $\partial D^{n}$ and $\psi^{-1}\left(\partial M_{0}\right)=\partial D^{n}$.

I claim $M_{0}$ is $(r+1)$-connected. Assuming this for a moment, then our result (a) follows at once from the main result of Haefliger [6], since this implies that $\psi$ is homotopic to an embedding, the homotopy being fixed near $\partial D^{n}$. Extend this 
homotopy to a homotopy of $f$ by letting it be fixed on $V_{0}$, and the result is an embedding. As for our result (b), this follows in exactly the same manner from the main result of Hudson [10], which shows that $\psi$ is homotopic to a piecewise linear embedding, the homotopy fixed near $\partial D^{n}$.

Hence we must only show that $M_{0}$ is $(r+1)$-connected. $M$ is 1-connected and $m \geqq n+3$ (unless $n=1, m=3$, which is trivial) so $M-V_{0}$ and thus $M_{0}$ is 1 -connected. So we show $H_{q}\left(M_{0}\right)=H_{q}\left(M-V_{0}\right)=0$ for $0<q \leqq r+1$, or by duality, we show $H_{c}^{m-q}\left(M-V_{0}\right)=0$ (compact supports), for $0<q \leqq r+1$.

Now we have the exact sequence:

$$
H_{c}^{m-q}(M) \leftarrow H_{c}^{m-q}\left(M-V_{0}\right) \leftarrow H_{c}^{m-q-1}\left(V_{0}\right) .
$$

Now since $H_{c}^{m-q}(M)=0$ for $0<q \leqq r+1$ ( $M$ being $(r+1)$-connected) and since $H_{c}^{n-q}\left(V_{0}\right)=0$ for $q \leqq[(r+1) / 2]$, which implies $H^{m-q-1}\left(V_{0}\right)=0$ for $0<q \leqq r+1$, the result follows. This completes the proof of 3.5.

We might point out that in $3.5(\mathrm{~b})$ the condition $m \geqq(4 / 3)(n+1)$ can be removed and a more complicated connectivity assumption made on $V$. A perusal of [10] will readily enable the reader to construct this. We now intend to show just how close the invariant $\gamma(f)$ comes to being an actual obstruction to getting an embedding.

3.6. Proposition. Let $f: V^{n} \rightarrow M^{m}$ be a continuous map of h-oriented manifolds ( $h$ a multiplicative theory as in Chapter II) with $V$ closed. If $\gamma(f) \neq 0$ then $f$ is not homotopic to any embedding whose normal bundle has a cross section.

Proof. If $f$ is an embedding with cross section then $\tilde{\chi}(f)=\varnothing$, so $\gamma(f)=j_{*}[\tilde{\chi}(f)]=0$ by 2.10 .

IV. Applications. In this section we apply the above methods to various special cases, most of which lay beyond the reach of previous methods. We would first like to present, however, without proof, the relative version of the main theorem. Its proof is completely analogous to 3.5 and we only give the stable range version of it.

4.1. TheOREM. Let $f:\left(V^{n}, \partial V\right) \rightarrow\left(M^{m}, \partial M\right)$ be an immersion whose normal bundle admits a cross section. Suppose $V$ is compact, and that $V, \partial V, M$ and $\partial M$ are $[(r+1) / 2],[r / 2],(r+1)$ and $r$-connected respectively, and that the map ${ }^{s} \tilde{\Omega}_{r}(\partial V)$ $\rightarrow{ }^{s} \tilde{\Omega}_{r}(V)$ is zero, $s=[(r+1) / 2]$. Finally assume $\gamma(f)=0$ over $s \Omega$, and $m \geqq(3 / 2)(n+1)$. Then $f$ is homotopic to an embedding, and the homotopy will be constant on $\partial V$ if $f \mid \partial V$ is an embedding.

We remind the reader that in the sequel, as before, $s$ denotes $[(r+1) / 2]$ and $r=2 n-m$.

4.2. THEOREM. Let $f: V^{n} \rightarrow M^{m}$ be a smooth immersion whose normal bundle has a cross section, where $V$ is compact and s-connected, and $M$ is $(r+1)$-connected. 
Suppose $f \mid V-p t$ is null homotopic. Then $f$ is homotopic to a smooth embedding if $m \geqq(3 / 2)(n+1)$ and a p.l. embedding if $m \geqq(4 / 3)(n+1)$.

Proof. We show $\tilde{\gamma}(f)=0$. Indeed, $\tilde{\gamma}(f)$ is the image of $[V]$ under the composition

$$
h_{n}(V) \stackrel{f_{*}}{\longrightarrow} h_{n}(M-p t) \stackrel{P}{\longrightarrow} h^{m-n}(M, p t) \stackrel{f^{*}}{\longrightarrow} h^{m-n}(V-p t) \stackrel{P}{\longrightarrow} h_{r}(V, p t) .
$$

Since $f \mid V-p t$ is null homotopic, $f^{*}$ above is zero, and so is $\tilde{\gamma}(f)$.

4.3. Corollary. For $m \geqq(3 / 2)(n+1)$, and $V$ as in $4.2, V^{n}$ embeds in $R^{m}$ provided g.d. $(V)<m-n$. (Here g.d. is the geometric dimension of the stable normal bundle. A converse to this theorem has been proved. See Handel [13].)

4.4. THEOREM. If $f: V^{n} \rightarrow M^{m-1}$ is a smooth immersion with $V$ compact and $s$-connected, and $M(r+1)$-connected, then $f$ is homotopic to a smooth embedding in $M \times R$.

Proof. If $i: M \times 0 \rightarrow M \times R$ is inclusion, $\nu($ if $)$ clearly has a cross section. So we show $\gamma($ if $)=0$. Let $S=(-\infty,-1] \cup[1, \infty)$, then $\gamma($ if $)$ is the image of $[V]$ under

$$
h_{n}(V) \rightarrow h_{n}(M \times(R-S)) \rightarrow h^{m-n}(M \times R, M \times S) \rightarrow h^{m-n}(V) \rightarrow h_{r}(V) .
$$

Since (if) is homotopic to a map with image in $M \times S,(\text { if })^{*}=0$ above.

We close with a few more specific examples.

(A) Suppose $V^{n}=S^{p} \times S^{n-p}, s<p \leqq n-p$, and $M$ is $(r+1)$-connected. We study the embedding problem in this case. If $f$ represents an element in $[V, M]$, and $m>n$, we can assume $f\left(S^{p} \times S^{n-p}\right) \subset M-p t$. The Hopf construction [9] on this yields a map $F: S^{m+1} \rightarrow S(M-p t)$, giving an element of $\Pi_{n}(M-p t)$, and thence, via the Hurewicz map, an element $\zeta$ in $h_{n}(M-p t)$, where $h$ is any theory relative to which $M$ is oriented. Let $v$ in $h^{m-n}(M, p t)$ be the dual of $\zeta$. Let $i_{1}: S^{p} \rightarrow S^{p} \times S^{n-p}$ and $i_{2}: S^{n-p} \rightarrow S^{p} \times S^{n-p}$ be the inclusions.

4.5. Proposition. Suppose g.d. $f^{*}(\tau M)<m-n$, and also $\left(f i_{1}\right)^{*} v=0=\left(f i_{2}\right)^{*} v$. Then $f$ is homotopic to a smooth embedding if $m \geqq(3 / 2)(n+1)$ and a p.l. embedding if $m \geqq(4 / 3)(n+1)$.

Proof. The geometric dimension condition insures $f$ is homotopic to an immersion with a cross section by [7]. So we show $\tilde{\gamma}(f)=0$. Now $\zeta=f_{*}[V]$, and $S^{p} \times S^{n-p}-p t \simeq S^{p} \vee S^{n-p}$. Also $\tilde{\gamma}(f)$ is given by

$$
h_{n}(V) \longrightarrow h_{n}(M-p t) \longrightarrow \tilde{h}^{m-n}(M) \stackrel{f^{*}}{\longrightarrow} h^{m-n}(V-p t) \longrightarrow h_{r}(V, p t) .
$$

Since $f^{*}$ factors through $\tilde{h}^{*}\left(S^{p} \times S^{n-p}-p t\right)=\tilde{h}^{*}\left(S^{p} \vee S^{n-p}\right)=\tilde{h}^{*}\left(S^{p}\right) \oplus \tilde{h}^{*}\left(S^{n-p}\right)$, we see that $f^{*} P^{-1} f_{*}[V]=f^{*}(v)=0$ provided $\left(f i_{1}\right)^{*} v=\left(f i_{2}\right)^{*} v=0$.

EXAMPLES. (1) Consider $\left[S^{5} \times S^{2}, \mathrm{HP}^{2} \times S^{4}\right]$. Here $r=2, s=1, m-n=5$. For any $f$ in this set one easily computes that g.d. $f^{*}(\tau M)<5$. To apply 4.5 note $f i_{2} \simeq 0$, so $\left(f i_{2}\right)^{*} v=0$. Also $\left(f i_{1}\right)^{*}$ factors through ${ }^{1} \widetilde{\Omega}^{5}\left(\mathrm{HP}^{2} \vee S^{4}\right)={ }^{1} \widetilde{\Omega}^{5}\left(\mathrm{HP}^{2}\right) \oplus{ }^{1} \widetilde{\Omega}^{5}\left(S^{4}\right)$ 
$={ }^{1} \widetilde{\Omega}^{5}\left(\mathrm{HP}^{2}\right)={ }^{1} \Omega_{3}\left(\mathrm{HP}^{2}\right)={ }^{1} \Omega_{3}(p t)$ since $\mathrm{HP}^{2}$ is 3 -connected. Now ${ }^{1} \Omega_{*}$ is just oriented cobordism, and hence ${ }^{1} \Omega_{3}(p t)=0$ by [16]. Thus $\left(f i_{1}\right)^{*} v=0$ and $\tilde{\gamma}(f)=0$.

(2) Consider $\left[S^{3} \times S^{13}, S^{11} \times V_{10,2}\right]$ where $V_{10,2}$ means all 2-frames in 10 space. $\left(f i_{1}\right)^{*} v=0$ since ${ }^{2} \Omega^{12}\left(S^{3}\right)=0$. Consider $\left(f i_{2}\right)^{*} v$. Since $\pi_{13}\left(V_{10,2}\right)=0, f i_{2}$ factors through $S^{11} \times *$, but ${ }^{2} \Omega^{12}\left(S^{11}\right)=0$, so $\left(f i_{2}\right)^{*} v$ and $\tilde{\gamma}(f)$ are 0 , and every element in this set of maps is homotopic to an embedding.

(B) Now let $V^{n}$ be $s$-connected and compact; this time we will restrict $M^{m}$ to be a sphere bundle over $S^{q}, \pi: M \rightarrow S^{q}$, with a cross section, $\sigma: S^{q} \rightarrow M$, where $r+1<q, r+1<m-q$. We write $S^{q} \vee S^{m-q}$ for $\sigma\left(S^{q}\right) \cup i\left(S^{m-q}\right)$, where $i: S^{m-q} \rightarrow M$ is the fiber inclusion over the base point. Thus $M-S^{q} \vee S^{m-q}$ is an open $m$-cell.

If $f$ represents a class in $[V, M]$ and $m>n$, we can thus assume that $f(V) \subset S^{q} \vee S^{m-q}$, and projection on each factor thus gives us two maps, $f^{1}: V \rightarrow S^{q}$ and $f^{2}: V \rightarrow S^{m-q}$. Let $\mu_{1}$ in ${ }^{s} \tilde{\Omega}^{q}\left(S^{q}\right)$ and $\mu_{2}$ in ${ }^{s} \tilde{\Omega}^{m-q}\left(S^{m-q}\right)$ be the standard generators. Note that $V$ and $M$ are ${ }^{s} \Omega$-orientable, as is the disc bundle associated with the bundle $M$. Let $v_{i}, i=1,2$, be the dual of $f_{*}^{i}([V])$. For any connected space $X$ we write $\alpha \cdot x$ (resp. $x \cdot \alpha)$ for the left (resp. right) action of ${ }^{s} \Omega^{*}(p t)$ on ${ }^{s} \Omega^{*}(X)$, and we write

$$
\rho: s \Omega *(X) \rightarrow{ }^{s} \Omega *(p t)
$$

for the map induced by $p t \rightarrow X$. The following proposition is a simple exercise in the Thom isomorphism, the Gysin sequence and Poincaré duality. It is much like previous calculations and we leave its proof to the reader.

4.6. Proposition. With the notation as above (and $m>n$ ), the dual of $\tilde{\gamma}(f)$ in ${ }^{s} \widetilde{\Omega}^{m-n}(V)$ is given by $\rho\left(v_{2}\right) \cdot f^{1^{*}}\left(\mu_{1}\right)+(-1)^{q(m-q)} \rho\left(v_{1}\right) \cdot f^{2^{*}}\left(\mu_{2}\right)$.

EXAMPLES. (1) Let $M=S^{q} \times S^{m-q}$, and suppose g.d. $(V)<m-n$ with connectivity assumptions as above. Then $f=f^{1} \times f^{2}$. If either $f^{1}$ or $f^{2}$ is stably null homotopic, then 4.6 shows that $f$ is homotopic to an embedding, provided $m \geqq(3 / 2)(n+1)$. Similarly, if $q=m-q, q$ odd, and $f^{1}, f^{2}$ represent elements in $\Pi^{*}(V)$ which agree up to sign at least, then again $\tilde{\gamma}(f)=0$ and $f$ is homotopic to an embedding for $m \geqq(3 / 2)(n+1)$. Similarly for p.l. embeddings if $m \geqq(4 / 3)(n+1)$.

(2) Consider the embedding problem when $V=S^{p} \times S^{n-p}$ and $M=S^{q} \times S^{m-q}$, where $n-p \geqq p>s, m-q \geqq q>r+1, m \geqq(4 / 3)(n+1)$. If $p \geqq q-1$, then $V$ is $r$-connected, $\tilde{\gamma}(f)=0$ and every map embeds; so we take the case where $p<q-1$. In this case, $\left[V, S^{q}\right]$ is in one-to-one correspondence with $\pi_{n-p}\left(S^{q}\right) \times \pi_{n}\left(S^{p}\right)$, and similarly for $\left[V, S^{m-q}\right]$. Thus a map from $S^{p} \times S^{n-p}$ into $S^{q} \times S^{m-q}$ yields, in the stable groups, an element in $\Pi_{n-p-q} \times \Pi_{n-q} \times \Pi_{n-p-m+q} \times \Pi_{n-m+q}$. If $f^{1}, f^{2}, i_{1}, i_{2}, v_{1}, v_{2}, v$ are as above in 4.5 and 4.6 , then this element is given by

$$
f \rightarrow\left[f^{1} i_{2}\right] \times v_{1} \times\left[f^{2} i_{2}\right] \times v_{2} .
$$

Since $f i_{1}$ is null homotopic we see from 4.5 that $\tilde{\gamma}(f)=0$ iff $\left(f i_{2}\right)^{*} v=0$. Working over stable cohomotopy theory (which is permissible by 2.15 ) we see from 4.6 that $\left(f i_{2}\right)^{*} v$, in $\Pi^{m-n}\left(S^{n-p}, p t\right)=\Pi_{r-p}$, is none other than

$$
v_{2} \circ\left[f^{1} i_{2}\right]+(-1)^{q(m-q)} v_{1} \circ\left[f^{2} i_{2}\right]
$$


In the dimensions where Toda's calculations are available, this quantity is easily calculated from [15, pp. 186-190]. Thus we can enumerate those maps which are homotopic to embeddings. For example:

(i) $\left[S^{4} \times S^{6}, S^{6} \times S^{10}\right]$ is in 1-1 correspondence with $Z \times Z$. Those maps $f$ such that $f^{2}$ or $f^{1} i_{2}$ is null homotopic are homotopic to embeddings. The others are not homotopic to embeddings with cross sections.

(ii) $\left[S^{10} \times S^{17}, S^{16} \times S^{26}\right]$ is in 1-1 correspondence with $Z_{504} \times Z_{2} \times Z_{2}$. Of these, 504 are not homotopic to embeddings with cross sections, and the other 1512 are homotopic to embeddings.

\section{BIBLIOGRAPHY}

1. A. L. Blakers and W. S. Massey, The homotopy groups of a triad. II, Ann. of Math. (2) 55 (1952), 192-201. MR 13, 485.

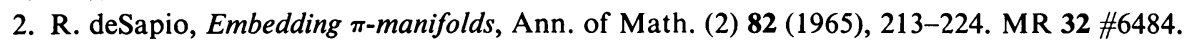

3. A. Dold, Relations between ordinary and extraordinary homology notes, Aarhus Colloquium on Topology, 1962, p. 2.

4. E. Dyer, Generalized cohomology theories, Mimeographed Notes, University of Chicago, Chicago, Ill.

5. A. Haefliger, Plongements différentiables dans le domaine stable, Comment. Math. Helv. 37 (1962/63), 155-176. MR 28 \#625.

6. - Plongements différentiables des variétés dans variétés, Comment. Math. Helv. 36 (1961), 47-82. MR 26 \#3069.

7. M. Hirsch, Immersions of manifolds, Trans. Amer. Math. Soc. 93 (1959), 242-276. MR 22 \#9980.

8. M. Hirsch and E. Zeeman, Engulfing, Bull. Amer. Math. Soc. 72 (1966), 113-115. MR 32 \#1718.

9. H. Hopf, Uber die Abbildungen von Spharen auf Spharen niedriger Dimension, Fund. Math. 25 (1935), 427.

10. J. F. P. Hudson, Piecewise linear embeddings, Ann. of Math. (2) 85 (1967), 1-31. MR 35 \#6149.

11. M. Kervaire and J. Milnor, Groups of homotopy spheres. I, Ann. of Math. (2) 77 (1963), 504-537. MR $26 \# 5584$.

12. R. Lashof and S. Smale, Self-intersections of immersed manifolds, J. Math. Mech. 8 (1959), 143-157. MR 21 \#332.

13. D. Handel, On the normal bundle of an embedding, Topology 6 (1967), 65-68. MR 35 \#3684.

14. R. Thom, Quelques propriétés globales des variétés différentiables, Comment. Math. Helv. 28 (1954), 17-86. MR 15, 890.

15. H. Toda, Composition methods in homotopy groups of spheres, Ann. of Math. Studies, no. 49, Princeton Univ. Press, Princeton, N. J., 1962. MR 26 \#777.

16. C. Wall, Determination of the cobordism ring, Ann. of Math. (2) 72 (1960), 292-311. MR $22 \# 11403$.

17. R. Wells, Modifying intersections, Illinois J. Math. 11 (1967), 389-403. MR 36 \#896.

18. G. W. Whitehead, Generalized homology theories, Trans. Amer. Math. Soc. 102 (1962), 227-283. MR 25 \#573.

Haverford College,

Haverford, Pennsylvania 19041 